\title{
Valorisation of Potato (Solanum tuberosum) Peel Waste: Extraction of Fibre, Monosaccharides and Uronic Acids
}

\author{
Regiane Scharf ${ }^{1,4} \cdot$ Rongchun Wang $^{1,2} \cdot$ Joanne Maycock ${ }^{1} \cdot$ Peter Ho $^{1} \cdot$ Shiguo Chen $^{3} \cdot$ Caroline Orfila $^{1}(\mathbb{D}$
}

Received: 26 July 2018 / Accepted: 26 November 2018 / Published online: 11 December 2018

(c) The Author(s) 2018

\begin{abstract}
Purpose The food and starch industries generate large quantities of potato peel waste (PPW) that can be exploited for a range of biotechnological and biofuel applications. The purpose of this study was to characterise the ultrastructure and monosaccharide composition of PPW.

Methods The ultrastructure of PPW was observed using light and immunofluorescence microscopy. Fibre was prepared from PPW using mild detergent and subjected to sequential acid hydrolysis followed by high performance anion exchange chromatography with pulsed amperometric detection (HPAEC-PAD).

Results Potato peels are composed of small, stacked cells which generally lack starch granules. These cells are surrounded by thick cell walls rich in pectic polysaccharides. Following sequential extraction, seven different sugars and two uronic acids were identified including (in order of abundance) mannose, galacturonic acid, xylose, glucose, fucose, glucuronic acid, galactose, rhamnose and arabinose. The monosaccharides and uronic acid products showed good stability in the acidic conditions during storage, which would facilitate their downstream purification and eventual commercialisation.
\end{abstract}

Conclusion PPW is a readily available source of fibre, monosaccharides and uronic acids.

\section{Graphical Abstract}

Potato peel waste

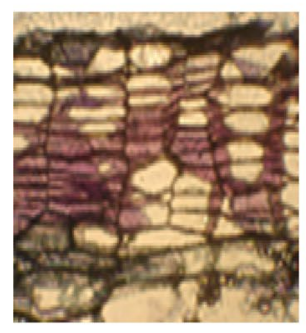

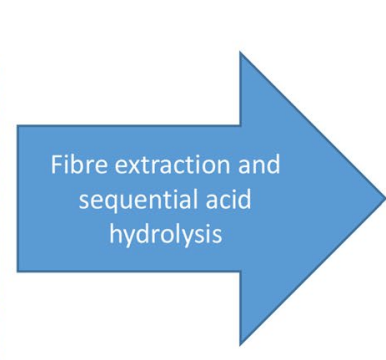

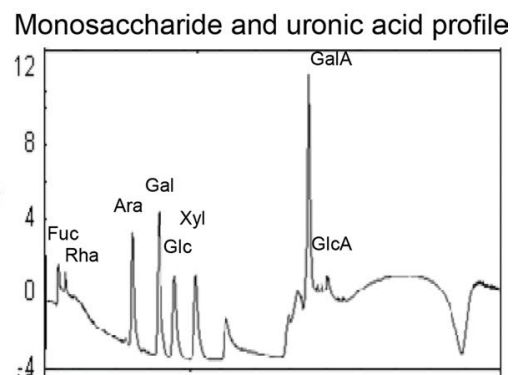

Keywords Potato peel waste $\cdot$ Solanum tuberosum $\cdot$ Fibre $\cdot$ Cell wall monosaccharide $\cdot$ Uronic acid $\cdot$ Sequential acid hydrolysis

\section{Statement of Novelty}

The monosaccharide and uronic acid composition of potato peel waste, an abundant by-product of the agri-food industry, has been characterised for the first time.

Caroline Orfila

c.orfila@leeds.ac.uk

Extended author information available on the last page of the article

\section{Introduction}

Worldwide potato tuber production has been increasing steadily due to rising demand for human nutrition and bioprocessing in China, Asia and Africa. It is now the fourth most produced edible crop after rice, maize and wheat, with an estimated annual yield of 370 thousand tonnes in 2013, of which between $20-30 \%$ by weight can be discarded during post-harvest processing. The potato processing industry 
generates large quantities of unavoidable potato peel waste (PPW) which can pose an environmental burden on both land and water resources due to rapid microbial degradation. Several technologies have been applied to recycle and valorise PPW [1-3]. These include ultrasound, high-pressure processing, sub-critical water extraction, acidic and enzymatic hydrolysis. These have been applied to obtain valuable components from PPW including biopolymers, polyphenols, glycoalkaloids and reducing sugars. The sugars are often used as feedstock for microbial fermentation in bioethanol production $[4,5]$. However, the cell wall monosaccharide composition of the extracts has not been previously investigated. It is likely that PPW is a source of a variety of monosaccharides and uronic acids which are themselves valuable products for commercialisation or further processing.

Therefore, the aims of this study were to (1) characterise the structure of PPW and (2) prepare a fibre extract from PPW and to sequentially extract sugars and uronic acids. The monosaccharides and uronic acids were characterised by high performance anion exchange chromatography with pulsed aerometric detection (HPAEC-PAD) which can identify and quantify all cell wall sugars in one chromatographic run. The storage stability the hydrolysis products was evaluated and the potential of PPW as a source of valuable fibre and sugars is discussed.

\section{Materials and Methods}

\section{Sampling of PPW}

Potato plants (Solanum tuberosum) from different cultivars Maris Piper, Russet Burbank and Lady Rosetta were used. Tubers were harvested and prepared the next day. The potatoes were peeled using a domestic OXO Good Grips Swivel Peeler; the thickness of the slices was measured using a Mercer England Thickness gage. The peels referred to here as PPW were immediately frozen and lyophilised.

\section{Microscopy of Peel Samples}

Fresh peel specimens $\left(0.5 \mathrm{~cm}^{3}\right)$ were fixed in $4 \%$ formaldehyde in PEM buffer (50 mM Pipes, $5 \mathrm{mM} \mathrm{MgSO}_{4}$ and 10 mM EGTA, pH 6.9). Fixative was removed with PEM and the samples were washed with phosphate buffered saline (PBS), dehydrated in an ethanol series (30-70\%) and embedded in Steadman wax (9:1 polyethylene glycol 400 distearate and 1-hexadecanol). Wax embedded samples were sectioned using a microtome with metal blade at $11^{\circ}$ and $50 \mu \mathrm{m}$ thickness. Sections were placed onto polysine-coated glass slides, followed by dewaxing with ethanol series (97-50\%). Sections were then stained with $0.1 \%$ Toluidine Blue for $10 \mathrm{~min}$, washed for $10 \mathrm{~min}$ in PBS, mounted with anti-fading glycerol phosphate buffered solution (Citifluor AF1, Agar Scientific, UK) and covered with a glass cover slip. Observations were made with a $\mathrm{BH} 2$ Olympus microscope. For immunofluorescence labelling of pectin, sections were incubated with $150 \mu \mathrm{L}$ of $3 \%(\mathrm{w} / \mathrm{v})$ milk protein in PBS for $1 \mathrm{~h}$ to reduce nonspecific binding. Monoclonal antibodies JIM5 and JIM7 which recognize different patterns of methyl esterification on homogalacturonan were kindly provided by Professor Paul Knox (Centre for Plant Sciences, University of Leeds, UK). The sections were incubated overnight at $4{ }^{\circ} \mathrm{C}$ in primary antibody (either JIM5 or JIM7), diluted 1:5 in PBS with milk. Control sections were incubated in PBS alone. Samples were washed twice with $0.1 \%$ v/v Tween 20 in PBS for $10 \mathrm{~min}$. All sections were incubated at room temperature for $1 \mathrm{~h}$ in secondary antibody (anti-rat goat $\operatorname{IgG}$ conjugated to FITC, Sigma), diluted 1:100 in PBS with milk. Samples were washed $10 \mathrm{~min}$ with $0.1 \% \mathrm{v} / \mathrm{v}$ Tween 20 in PBS plus $10 \mathrm{~min}$ in PBS. Samples were then stained with $0.1 \%$ Toluidine Blue for $10 \mathrm{~min}$ to reduce autofluorescence interference, washed for $10 \mathrm{~min}$ in PBS, mounted with antifading glycerol phosphate buffered solution (Citifluor AF1, Agar Scientific, UK) and covered with a glass cover slip. Observations were made with a Confocal Zeiss Axioplan Imaging LSM 510 Meta.

\section{Preparation of Fibre from PPW}

Lyophilised PPW (10 g) was homogenised using a homogeniser (Ultra Turrax, IKA, Staufen, Germany) at 22,700 g with $50 \mathrm{~mL}$ of cold mixed-cation buffer (MCB) $(10 \mathrm{mM}$ $\mathrm{NaOAC}, 3 \mathrm{mM} \mathrm{KCl}, 2 \mathrm{mM} \mathrm{MgCl} \mathrm{Mn}_{2}$ and $1 \mathrm{mM} \mathrm{CaCl}_{2}, \mathrm{pH}$ 6.5) containing Triton $\mathrm{X}-100(2 \mathrm{mg} / \mathrm{ml})$ for $5 \mathrm{~min}$ at $4{ }^{\circ} \mathrm{C}$. The detergent suspension was removed by washing through a metal sieve $(45 \mu \mathrm{m})$ with $100 \mathrm{~mL}$ of chilled MCB without Triton X-100. This step removed intact starch granules that could recovered by sedimentation. The pellet was washed with $2 \mathrm{~L}$ of water and finally with $10 \mathrm{~mL}$ of $50 \%$ acetone to remove moisture. After washing, the fibre material was dried overnight in an oven at $35^{\circ} \mathrm{C}$.

\section{Sequential Hydrolysis of PPW Fibre}

Fibre hydrolysis was performed in duplicate in three sequential steps: firstly, $2 \mathrm{mg}$ of PPW fibre were hydrolysed with $1 \mathrm{~mL}$ of $0.1 \mathrm{M}$ trifluoroacetic acid (TFA) for $1 \mathrm{~h}$ at $100{ }^{\circ} \mathrm{C}$. Samples were centrifuged at $2800 \mathrm{~g}$ at $4{ }^{\circ} \mathrm{C}$ for $10 \mathrm{~min}$ and the supernatant was collected for analysis. Secondly, the solid residue was hydrolysed with $2 \mathrm{M}$ TFA for $1 \mathrm{~h}$. Tubes were centrifuged at $2800 \mathrm{~g}$ at $4{ }^{\circ} \mathrm{C}$ for $10 \mathrm{~min}$ and the supernatant was collected for analysis. Finally, the residue was hydrolysed with $1 \mathrm{M} \mathrm{H}_{2} \mathrm{SO}_{4}$ for $1 \mathrm{~h}$. TFA was removed using a centrifugal evaporator (Genevak, Surrey, UK). Dried samples were resuspended with $1 \mathrm{~mL}$ of milli-Q purified water 
and filtered using a $0.45 \mu \mathrm{m}$ nylon filter prior to monosaccharide and uronic analysis. $\mathrm{H}_{2} \mathrm{SO}_{4}$ was neutralised using $\mathrm{NaOH}$, and the sample diluted with milli-Q purified water prior to chromatographic analysis. Samples were kept chilled at all times and not exposed to bright light.

\section{Analysis of Monosaccharide Composition Using High Performance Anion Exchange Chromatography Amperometric Detection (HPAEC-PAD)}

The monosaccharide composition was determined by HPAEC-PAD using a method adapted from [6]. The column used was PA20 (Dionex, Thermo Scientific). Column temperature was $30{ }^{\circ} \mathrm{C}$, flow rate of $0.30 \mathrm{~mL} / \mathrm{min}$ and injection volume was $10 \mu \mathrm{L}$. The 65 min elution program consisted of linear gradient from $10 \mu \mathrm{M}$ to $5 \mu \mathrm{M} \mathrm{NaOH}$ from 0 to $1.5 \mathrm{~min}$, followed by isocratic elution with $5 \mu \mathrm{M}$ $\mathrm{NaOH}$ from 1.5 to 30 min, linear gradient up to $1 \mathrm{M} \mathrm{NaOH}$ from 30 to $40 \mathrm{~min}$, column washing with $1 \mathrm{M}$ from 40 to $45 \mathrm{~min}$, linear gradient to $10 \mathrm{mM}$ from 45 to $55 \mathrm{~min}$ following equilibration of the column with $10 \mathrm{mM} \mathrm{NaOH}$ from 55 to $65 \mathrm{~min}$. Monosaccharides were detected using a pulsed amperometric detector with gold working electrode and silver reference electrode. Monosaccharide standards were L-fucose, L-rhamnose, L-arabinose, D-galactose, D-glucose, D-xylose, D-mannose, D-galacturonic acid and D-glucuronic acid. D-Fructose was used as an internal standard. A standard mixture run was performed before sample analysis to determine response factor. The external standard method of calibration was used, with each curve prepared from seven different concentrations of standard solutions. The range of standards concentration used was from 0.30 to $100 \mu \mathrm{g} / \mathrm{mL}$. The external standard (fructose) concentration used was $200 \mu \mathrm{g} / \mathrm{mL}$.

Method validation for analysis of sugars was performed according to ICH recommendations and the European Commission Directive for the performance of analytical methods including linearity, precision and accuracy, which are principal components of quantification. Linearity was investigated by analysis of peak area response versus concentration over a range of $11 \mathrm{ng} / \mathrm{mL}$ to $150 \mu \mathrm{g} / \mathrm{mL}$. For calibration curves, the peak areas of the Dionex chromatogram were plotted against on-column amount and analysis was performed on two separate occasions with triplicate injections of each concentration. Precision and accuracy were evaluated for galactose at three quality control (QC) concentration $(25,50$ and $100 \mu \mathrm{g} / \mathrm{mL}$ ). The lower QC is representative of the lower concentration of hydrolysed sugars from $\mathrm{CW}$ samples, and the high QC level is near the upper boundary of the standard curve. Intra-day precision and accuracy was calculated from triplicate injection of the 3 concentrations on the same day. Inter-day precision and accuracy was determined by analysis of triplicate injections of the 3 concentrations on the 3 separate days. Values for precision are expressed as relative standard deviation (R.S.D) and relative error (R.E.) for accuracy.

To assess storage stability, hydrolysates and standards were stored for 3 days at $4{ }^{\circ} \mathrm{C}$ and re-analysed.

\section{Results and Discussion}

\section{Potato Peel Structure Investigated Using Microscopy}

Thin sections $(50 \mu \mathrm{m})$ were stained with toluidine blue to visualise the cellular organisation of potato skin, the main constituent of PPW (Fig. $1 \mathrm{a}-\mathrm{c}$ ). The micrographs show that for all varieties of potato, the skin consists of stacks of between 8 and 15 oblong cells measuring $40 \mu \mathrm{m}$ in length on the axis parallel to skin surface, by around $10 \mu \mathrm{m}$ in width on the axis perpendicular to skin surface. A clear layer of suberin is observed on the most external surface. There does not appear to be any starch granules within the skin cells, however are abundant in larger and rounder periderm cells underneath the skin (indicated with single arrow head on Fig. 1a). This is likely to be the main source of starch in PPW. Labelling of the same sections with the anti-pectin antibodies JIM5 and JIM7 showed abundance of pectin. Both methyl esterified (Fig. 1f) and non-methyl esterified (Fig. 1e) homogalacturonan were localised to cell walls of all cells in the skin and periderm. Mild autofluorescence (Fig. 1d) suggested the presence of cell wall phenolics. These micrographs suggest that potato peels are a rich source of cell wall material and pectin in particular.

\section{Preparation of Fibre from PPW}

The tightly packed cells of peel tissue make extraction of fibre difficult. To break open the cells and remove the lipids and proteins from cell membranes, as well as the suberin layer, PPW was homogenised in a cold buffer containing triton X-100, a non-ionic surfactant. This step allowed recovery of some intact starch granules. Even through starch is not visible in skin cells, it is our experience that periderm cells are often present in PPW. The yield of fibre was around 20-24\% (dry weight) of PPW.

\section{Chromatographic Separation of Cell Wall Monosaccharides and Uronic Acids}

We used HPAEC-PAD to separate eight sugars and two uronic acids in one chromatographic run (Fig. 2a; Table 1). Peak area varied linearly with on-column amount over the ranges used $(\mathrm{R}>0.98)$. Intra-day and inter-day precision was calculated for galactose as R.S.D. $<4.6 \%,<1.7 \%$ and $<6.4 \%$ for $25 \mu \mathrm{g} / \mu \mathrm{L}, 50 \mu \mathrm{g} / \mathrm{mL}$ and $100 \mu \mathrm{g} / \mathrm{mL}$ concentrations 

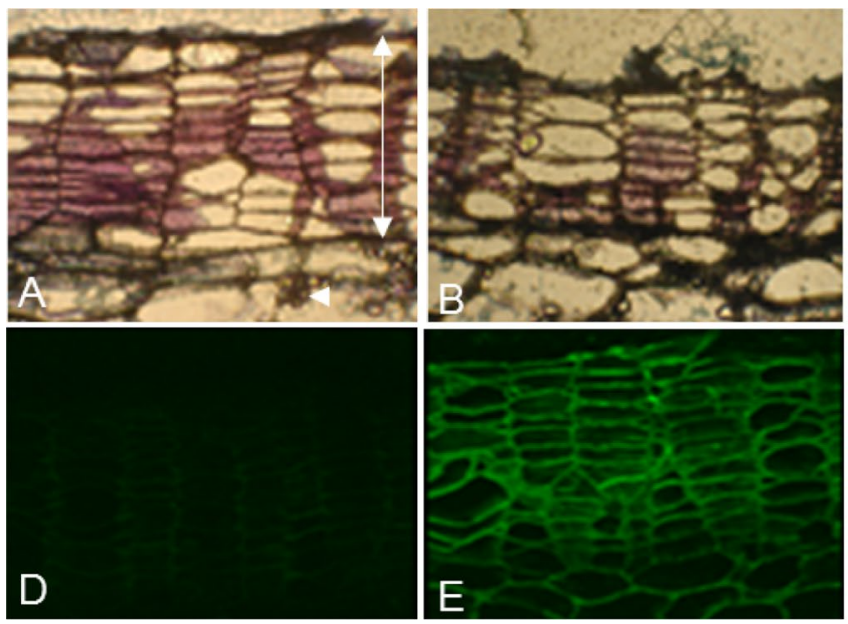

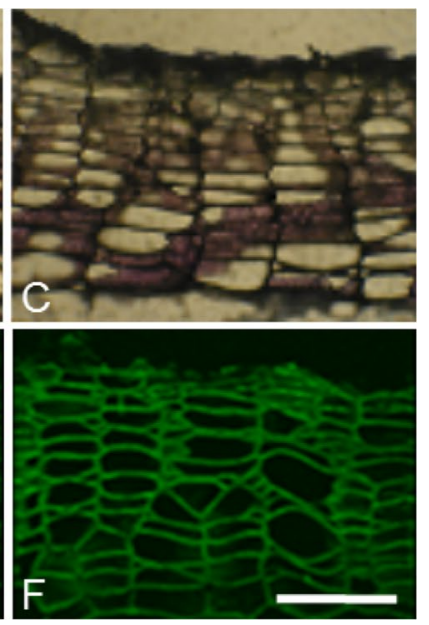

Fig. 1 Light and fluorescence microscopy of wax-embedded sections of potato tuber peel. Top row show toluidine blue stained sections of varieties Lady Rosetta (a), Maris Piper (b) and Russet Burbank (c). Double arrow shows skin tissue composed of stacked cells. Single arrow head shows presence of starch granules in periderm tis-

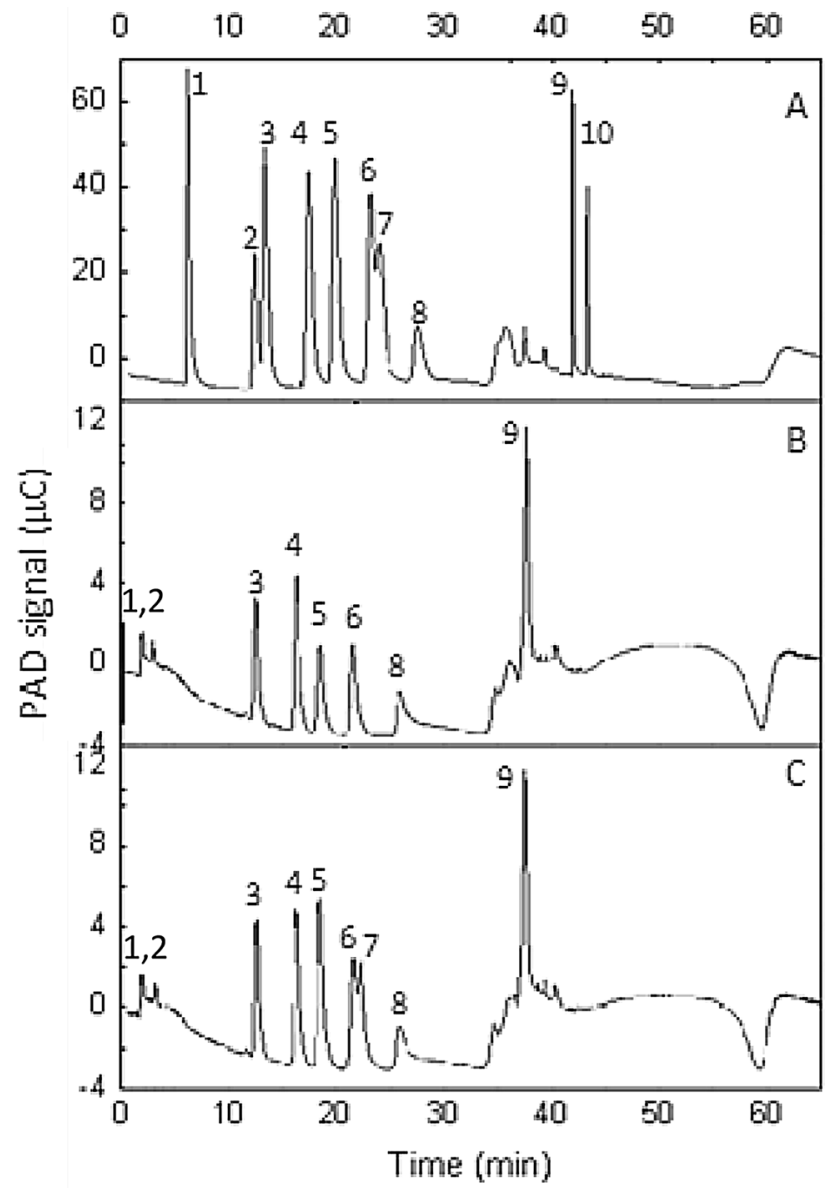

Fig. 2 HPAEC-PAD separation of a standards at $25 \mu \mathrm{g} / \mathrm{mL}$ b fibre hydrolysate and $\mathbf{c}$ fibre hydrolysate spiked with mannose. $\mathrm{mL}$ (1) fucose, (2) rhamnose, (3) arabinose, (4) galactose, (5) glucose, (6) xylose, (7) mannose, (8) fructose - used as internal standard (9) galacturonic acid and (10) glucuronic acid sue underneath skin. Bottom row shows immunofluorescence of skin (Maris Piper only) with no antibody (d, negative control), with JIM5 (e) and JIM7 (f) antibodies showing abundant and even presence of both methyl-esterified and non-esterified pectin. Scale bar $=50 \mathrm{um}$

Table 1 Chromatographic performance of eight monosaccharides and two uronic acids separated within the same chromatographic run using HPAEC-PAD

\begin{tabular}{llllll}
\hline Peak & Compound & $\begin{array}{l}\text { Rt }(\min ) \\
\text { Mean } \pm \mathrm{SD}^{\mathrm{a}}\end{array}$ & $\mathrm{RSD}(\%)^{\mathrm{b}}$ & $\begin{array}{l}\mathrm{LOQ}(\mathrm{ng} / \\
\mathrm{mL})\end{array}$ & $\mathrm{R}^{2}$ \\
\hline 1 & Fucose & $6.15 \pm 0.18$ & 2.88 & 47 & 0.9999 \\
2 & Rhamnose & $12.09 \pm 0.46$ & 3.79 & 47 & 0.9996 \\
3 & Arabinose & $13.10 \pm 0.45$ & 3.46 & 47 & 0.9999 \\
4 & Galactose & $16.81 \pm 0.63$ & 3.77 & 47 & 0.9997 \\
5 & Glucose & $19.09 \pm 0.72$ & 3.79 & 93 & 0.9993 \\
6 & Xylose & $22.26 \pm 0.81$ & 3.65 & 93 & 0.9998 \\
7 & Mannose & $23.08 \pm 0.83$ & 3.62 & 47 & 0.9999 \\
8 & Fructose & $26.62 \pm 1.06$ & 4.00 & 47 & 0.9999 \\
9 & Galacturonic & $41.80 \pm 0.05$ & 0.13 & 93 & 0.9823 \\
& acid & & & & 0.9999 \\
10 & Glucuronic & $43.10 \pm 0.03$ & 0.69 & 93 & \\
& acid & & & &
\end{tabular}

$\mathrm{n}=2,3$ repetitions each

$R t$ retention time, $S D$ standard deviation, $R S D$ relative standard deviation, $L O Q$ limit of quantification

respectively. Good intra-day and inter-day accuracy was demonstrated across the concentration range with relative error $<-7 \%$. The precision and accuracy meet performance criteria for analytical methods, with precision (R.S.D.) and accuracy (R.E) within 15\% (Table 1). All sugars and uronic acids were stable upon storage in acidic conditions for 3 days at $4{ }^{\circ} \mathrm{C}$, stability ranged between $95-100 \%$ (data not shown). Injection of hydrolysates shifted peaks to earlier retention times compared to standards in water (Fig. 2b), but all compounds were confirmed by spiking. Separation of xylose and 
Table 2 Yield percentage of monosaccharide and uronic acids released from PPW fibre after sequential hydrolysis

\begin{tabular}{lllll}
\hline \multicolumn{5}{c}{ Yield percentage $^{\mathrm{a}}$} \\
\cline { 2 - 5 } & $0.1 \mathrm{M}$ TFA & $2 \mathrm{M}$ TFA & $1 \mathrm{M} \mathrm{H}_{2} \mathrm{SO}_{4}$ & $\begin{array}{l}\text { Total } \\
\text { extractable }\end{array}$ \\
\hline Extract yield & 15 & 27 & 28 & 70 \\
Monos & & & & \\
Fuc & 0.65 & 1.72 & 0.90 & 3.27 \\
Rha & 1.49 & 0.98 & 0.00 & 2.47 \\
Ara & 2.12 & 0.14 & 0.08 & 2.34 \\
Gal & 1.49 & 0.95 & 0.12 & 2.56 \\
Glu & 0.52 & 1.24 & 1.80 & 3.56 \\
Xyl & 0.32 & 1.98 & 1.37 & 3.67 \\
Man & 0.00 & 0.00 & 7.84 & 7.84 \\
GalA & 1.15 & 0.55 & 2.31 & 4.01 \\
GlcA & 1.78 & 0.14 & 1.25 & 3.18 \\
Total monos & 9.50 & 7.72 & 15.68 & 32.89 \\
\hline
\end{tabular}

mannose was problematic but spiking of samples confirmed their separation even in fibre hydrolysates (Fig. 2c).

\section{Monosaccharide and Uronic Acid Composition of PPW Fibre Hydrolysates}

Sequential acid hydrolysis using increasingly stronger acid hydrolysed the fibre and extracted mixtures of sugars and uronic acids with different compositions. Table 2 shows the yield of the extracts and sugars extracted at each step. The analysis indicated that PPW contains polymers made of a variety of monosaccharides, including neutral sugars and uronic acids. A large proportion of arabinose, galactose, rhamnose and uronic acids was extracted with the mild TFA conditions $(0.1 \mathrm{M})$, indicating presence of branched rhamnogalacturonan I (RG I) that can be easily extracted and is acid labile. Meanwhile stronger acidic conditions extracted glucose, xylose, galactose and fucose, indicative of xyloglucans, as well as uronic acid and rhamnose, but little arabinose, indicating presence of galactan rich rhamnogalacturonan I. Finally, the strongest acid tested in this study (1 M $\mathrm{H}_{2} \mathrm{SO}_{4}$ ) extracted mannose, glucose, xylose and uronic acids, indicating presence of xyloglucans, glucomannans and pectin. The composition of the residue was not determined. This composition analysis shows that PPW contains mixture of biopolymers including pectins and hemicelluloses that can be easily hydrolysed using relatively mild acid conditions. The high arabinose content suggests that potato RGI may be higher in arabinan than previously reported [6].

\section{Conclusion}

This study demonstrated the potential of potato peel waste as a useful source of fibre, a variety of monosaccharide and uronic acid which could be further purified and commercialised.

Acknowledgements This work was funded through a $\mathrm{PhD}$ scholarship to RS by the Agricultural and Horticultural Development Board (AHDB) and the Yorkshire Agricultural Society. The authors wish to thank Professor Paul Knox (University of Leeds) for the gift of JIM5 and JIM7 antibodies, and Dr Mark Stalham (NIAB) for providing fresh potato tissue.

Open Access This article is distributed under the terms of the Creative Commons Attribution 4.0 International License (http://creativeco mmons.org/licenses/by/4.0/), which permits unrestricted use, distribution, and reproduction in any medium, provided you give appropriate credit to the original author(s) and the source, provide a link to the Creative Commons license, and indicate if changes were made.

\section{References}

1. Arapoglou, D., Varzakas, T., Vlyssides, A., Israilides, C.: Ethanol production from potato peel waste (PPW). Waste Manag. 30, 1898-1902 (2010)

2. Galhano Dos Santos, R., Ventura, P., Bordado, J.C., Mateus, M.M.: Valorizing potato peel waste: an overview of the latest publications. Rev. Environ. Sci. Bio/Technol. 15, 585-592 (2016)

3. Wu, D.: Recycle technology for potato peel waste processing: a review. Proc. Environ. Sci. 31, 103-107 (2016)

4. Khawla, B.J., Sameh, M., Imen, G., Donyes, F., Dhouha, G., Raoudha, E.G., Oumèma, N.-E.: Potato peel as feedstock for bioethanol production: a comparison of acidic and enzymatic hydrolysis. Ind. Crops Prod. 52, 144-149 (2014)

5. Pathak, P.D., Mandavgane, S.A., Puranik, N.M., Jambhulkar, S.J., Kulkarni, B.D.: Valorization of potato peel: a biorefinery approach. Crit. Rev. Biotechnol. 38, 218-230 (2018)

6. Obro, J., Harholt, J., Scheller, H.V., Orfila, C.: Rhamnogalacturonan I in Solanum tuberosum tubers contains complex arabinogalactan structures. Phytochemistry 65, 1429-1438 (2004)

Publisher's Note Springer Nature remains neutral with regard to jurisdictional claims in published maps and institutional affiliations. 


\section{Affiliations}

\section{Regiane Scharf ${ }^{1,4} \cdot$ Rongchun Wang $^{1,2} \cdot$ Joanne Maycock ${ }^{1} \cdot$ Peter Ho $^{1} \cdot$ Shiguo Chen $^{3} \cdot$ Caroline Orfila $^{1}$ (I)}

1 School of Food Science and Nutrition, University of Leeds, Leeds LS2 9JT, UK

2 School of Chemistry and Chemical Engineering, Harbin Institute of Technology, Harbin 150001, China

3 Zhejiang Key Laboratory for Agro-Food Processing, Department of Food Science and Nutrition, Fuli Institute of Food Science, Zhejiang University, Hangzhou 310058, China

4 Present Address: Nestlé Product Technology Centre (PTC), York YO31 8FY, UK 